\title{
Potent and sustained inhibition of HIF-1 $\alpha$ and downstream genes by a polyethyleneglycol-SN38 conjugate, EZN-2208, results in anti-angiogenic effects
}

\author{
Puja Sapra $\cdot$ Patricia Kraft $\cdot$ Fabio Pastorino $\cdot$ Domenico Ribatti $\cdot$ \\ Melissa Dumble $\cdot$ Mary Mehlig $\cdot$ Maoliang Wang $\cdot$ Mirco Ponzoni · \\ Lee M. Greenberger · Ivan D. Horak
}

Received: 18 November 2010/Accepted: 13 March 2011/Published online: 31 March 2011

(c) The Author(s) 2011. This article is published with open access at Springerlink.com

\begin{abstract}
Topoisomerase I inhibitors down-regulate HIF$1 \alpha$ leading to tumor growth inhibition, but only while maintaining sustained levels of drug exposure. EZN-2208, a multi-arm $40 \mathrm{kDa}$ pegylated, releasable SN38-drug conjugate, provides higher, longer lasting exposure of tumors to SN38 in contrast to SN38 that is released from CPT-11. EZN-2208 also consistently has greater antitumor activity than CPT-11 in a variety of solid and hematological tumor models. In this report, the ability of PEG-SN38 to downregulate HIF- $1 \alpha$ and its downstream targets, in a more potent, sustained manner compared with CPT- 11 was examined. To do so, U251 glioma xenografts that stably expressed a hypoxia response element-dependent luciferase reporter gene were implanted in mice. After treatment it was found that EZN-2208 induced potent, sustained HIF- $1 \alpha$ downregulation ( $37 \%$ at $48 \mathrm{~h}$ and $83 \%$ at $120 \mathrm{~h}$ ) in the tumors,
\end{abstract}

Electronic supplementary material The online version of this article (doi:10.1007/s10456-011-9209-1) contains supplementary material, which is available to authorized users.

P. Sapra - P. Kraft · M. Dumble - M. Mehlig $\cdot$ M. Wang .

L. M. Greenberger $(\square)$. I. D. Horak

Enzon Pharmaceuticals, 20 Kingsbridge Road,

Piscataway, NJ 08854, USA

e-mail: lee.greenberger@enzon.com

F. Pastorino $\cdot$ M. Ponzoni

Experimental Therapies Unit, Laboratory of Oncology,

G. Gaslini Children's Hospital, Genoa, Italy

D. Ribatti

Department of Human Anatomy, University of Bari, Bari, Italy

Present Address:

P. Sapra

Pfizer Inc., 401N Middletown Road, Pearl River, NY 10965, USA whereas CPT-11 caused only minor, transient HIF- $1 \alpha$ downregulation. In addition, EZN-2208 down-regulated mRNA levels of HIF- $1 \alpha$ targeted genes (MMP2, VEGF1, Glut1, Glut 3 and TGF $\beta 1$ ). Further, western blot analyses of xenograft tumors demonstrated that EZN-2208 had significantly more effect than CPT-11 in down-regulating HIF- $1 \alpha$, VEGF, Glut1 and MMP2 protein levels. Significant down-regulation of HIF- $1 \alpha$ and VEGF proteins translated to EZN-2208's superior anti-angiogenic activity compared with CPT-11, confirmed by microvessel density reduction in a chorioallantoic membrane assay and in CD-31 immunohistochemistry studies. Additional studies done with matrigel implants devoid of tumor cells show that EZN-2208 significantly inhibits angiogenesis while CPT-11 has little or no effect. It is concluded that the superior antitumor activity of EZN2208 compared with CPT-11 is attributed, in part, to an antiangiogenic effect. Ongoing clinical Phase I and Phase II studies will assess safety and efficacy of EZN-2208.

Keywords SN38 - Pegylation - HIF-1 $\alpha$ - Angiogenesis · CPT-11 (Camptosar)

$\begin{array}{ll}\text { Abbreviations } \\ \text { MTD } & \text { Maximum tolerated dose } \\ \text { HIF-1 } & \text { Hypoxia-inducible factor-1 } \\ \text { HRE } & \text { Hypoxia-responsive element } \\ \text { PEG } & \text { Polyethylene glycol } \\ \text { BL } & \text { Bioluminescence }\end{array}$

\section{Background}

Hypoxia-inducible factor 1 (HIF-1), a master regulator of transcriptional responses of mammalian cells to oxygen 
deprivation, plays a critical role in the expression of many genes that control angiogenesis, glucose metabolism, cell proliferation, cell survival, and metastasis in response to hypoxia [1,2]. Elevated expression of the alpha subunit of HIF-1 (HIF-1 $\alpha$ ) is associated with poor prognosis in many types of solid tumors such as lung, breast, colorectal, brain, pancreatic, ovarian, renal, and bladder cancers [3]. In addition, HIF-1 is involved in the up-regulation of downstream VEGF that exerts pro-angiogenic effects on the tumors' vascular endothelial cells, resulting in increased vascular permeability and neo-angiogenesis [4-7]. Since elevated HIF- $1 \alpha$ expression correlates with neo-angiogenesis, drug resistance and overall poor treatment outcome, compounds that inhibit HIF- $1 \alpha$ may have broad utility in the control of cancer $[2,8]$.

In recent years, strategies to identify direct and indirect inhibitors of HIF- $1 \alpha$ that function by blocking HIF- $1 \alpha$ expression levels and/or activity have been developed, including cell-based reporter screens, antisense approaches, targeting key protein-protein interactions, increasing HIF$1 \alpha$ protein turnover or utilizing HIF oligonucleotide decoy [9]. Topoisomerase I inhibitors, including camptothecin analogs and topotecan, were identified as HIF- $1 \alpha$ inhibitors in a high throughput cell-based HRE-luciferase reporter screen [10]. Melillo et al. demonstrated that topotecan inhibits HIF- $1 \alpha$, leading to a marked decrease in angiogenesis and to significant tumor growth inhibition [11, 12]. However, these effects were achieved only when the topisomerase I inhibitor (for example, topotecan) was administered on a daily schedule, and not when the drug was given on an intermittent schedule [11]. Based on these preclinical data, clinical strategies should aim at identifying the optimum dose and schedule of topoisomerase I inhibitors that would achieve continuous suppression of HIF-1.

Camptothecin-11 (CPT-11; Camptosar, irinotecan), is a topoisomerase I inhibitor that upon hydrolysis by carboxylesterase 2 yields the active moiety, SN38 (10-hydroxy-7ethyl-camptothecin). EZN-2208 is a pegylated-SN38 drug conjugate made by linking SN38 to a $40 \mathrm{kDa} 4$-arm polyethylene glycol (PEG) molecule [13] (Fig. 1, supplement). Upon addition to plasma, SN38 is released, thereby mediating the cytotoxic action of the compound. We have previously shown in preclinical xenograft models (including an in vivo CPT-11 resistance model), that EZN-2208 has a significantly enhanced efficacy compared with CPT-11 when both the drugs were compared at their respective maximum tolerated doses [14-16]. We have also shown that when compared at their respective MTDs, EZN-2208 provides higher and longer lasting exposure of tumors to SN38 via preferential accumulation of EZN-2208 in the solid tumors (enhanced permeability and retention effect) compared with CPT-11 [15], which could explain improved efficacy of EZN-2208 over CPT-11. Thus, we hypothesized that, due to favorable pharmacokinetic and biodistribution properties of EZN-2208, this molecule will work as a sustained delivery system for SN38 and, therefore, will down-regulate HIF- $1 \alpha$ in a more potent and sustained manner compared to CPT- 11 . In this report, we evaluate this hypothesis using the U251-HRE xenograft model system [10]. We demonstrate that sustained SN38 delivery by EZN-2208 down-modulates HIF- $1 \alpha$ protein, down regulates the expression of proteins controlled by HIF- $1 \alpha$, in particular VEGF, and has more anti-angiogenic activity compared to CPT- 11 .

\section{Materials and Methods}

Xenografts and Bioluminescence imaging

U251-HRE xenografts (cells kindly provided by Dr. Melillo, $\mathrm{NIH}$, Rockville, MD) were established in the flanks of female nude mice by injecting $1 \times 10^{7}$ cells. When tumors reached $100 \mathrm{~mm}^{3}$, the mice received EZN-2208 or CPT-11 at their respective MTDs (single or multiple dosing regimen). Luciferase expression in the U251-HRE tumors was measured using bioluminescence (BL) (Xenogen IVIS 100 Imaging Station, Xenogen Corp.) following firefly D-luciferin $(150 \mathrm{mg} / \mathrm{kg}$, IP) injection at 0,48 and $120 \mathrm{~h}$ from start of treatment. Because treatment reduced tumor mass (data not shown), BL values (total flux/photon/second, determined using the Xenogen Live ${ }^{\circledR}$ Image Analysis software) were normalized for tumor mass. The percent of tumor BL/mg tumor mass remaining at each time point, relative to the zero-time baseline for each respective treatment group, was calculated. It is important to note that the doses of EZN-2208 stated in this article refer to $\mathrm{SN} 38$ equivalents. For example, a dose of $30 \mathrm{mg} / \mathrm{kg}$ EZN2208 means that the dose contains $30 \mathrm{mg} / \mathrm{kg}$ of SN38 and $870 \mathrm{mg} / \mathrm{kg}$ (29-fold higher) of the whole conjugate, assuming that the loading of SN38 in the whole EZN-2208 is 3.45\%.

Relative quantification RT-PCR mRNA analysis and Western Blot protein analysis

Tumors were collected from U251-HRE xenografts at 48 and $120 \mathrm{~h}$ after receiving a single intravenous MTD treatment of either EZN-2208 or CPT-11. Total cellular RNA was extracted using Ambion RNAqueous kit and converted to cDNA using a high capacity cDNA reverse transcription kit (Applied Biosystems). HIF-1 $\alpha$, Glut1, Glut3, VEGF1, TGFBR 1 and MMP2 mRNA were measured individually by TaqMan gene expression and normalized by co-amplification of the reference gene, 18S rRNA. Results were quantitated with RQ Manager Software analysis. Differences between effects were determined by unpaired, two-tailed $t$ test with Welch correction. For protein analysis, tumors (120 h samples, 1-5 mg) were lysed in SDS-PAGE sample 


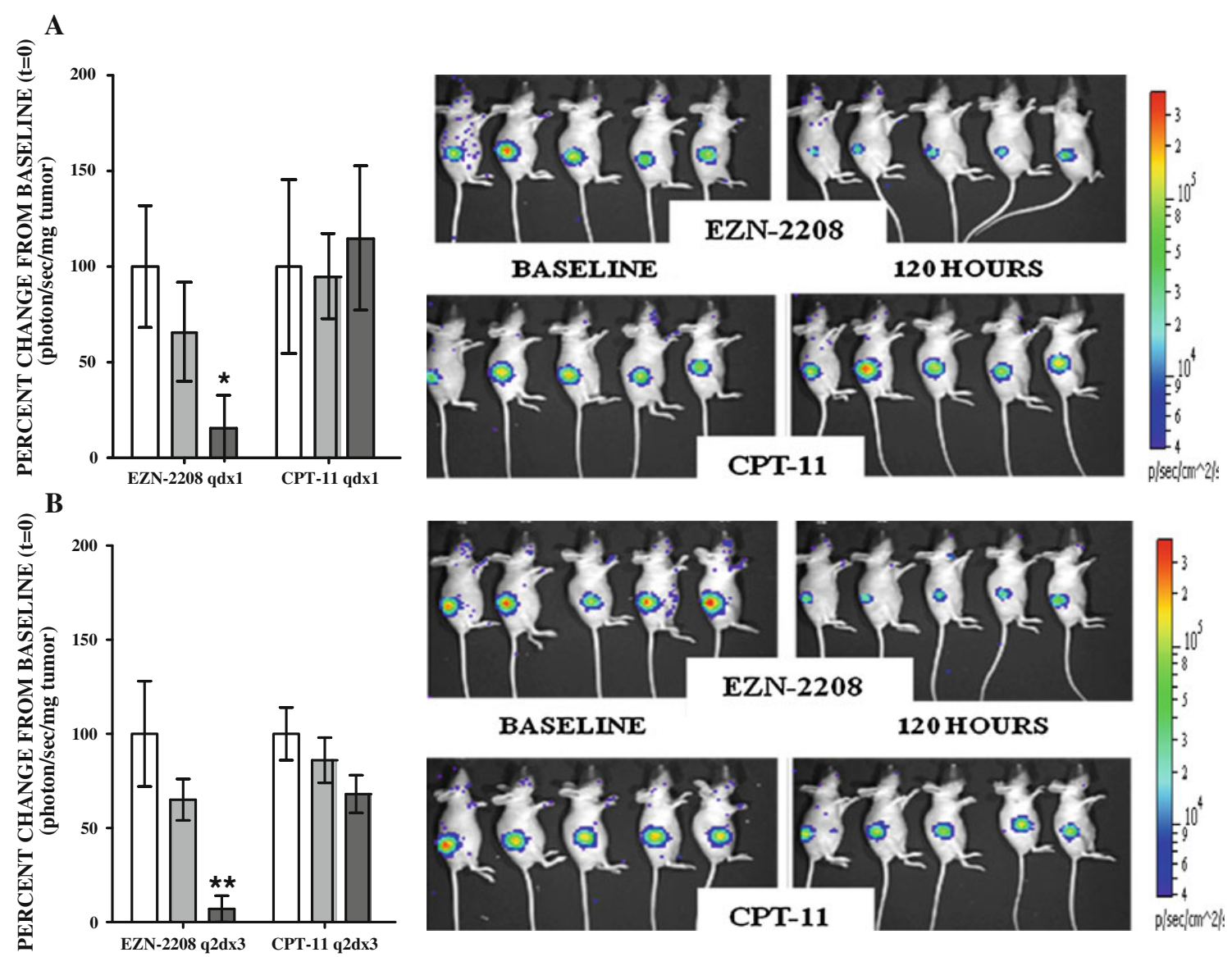

Fig. 1 HIF- $1 \alpha$ dependent luciferase expression inhibition in a U251HRE glioblastoma xenograft following treatment with EZN-2208 or CPT-11 using a single or $\mathbf{b}$ multiple dosing regimes. A Single, IV, qdx 1 treatment of EZN-2208 (30 mg/kg) or CPT-11 (80 mg/kg). $B$ Multiple, IV, q2dx3 treatment of EZN-2208 $(10 \mathrm{mg} / \mathrm{kg})$ or CPT-11 $(40 \mathrm{mg} / \mathrm{kg})$.

buffer. Each sample (10 $\mu \mathrm{g}$ total proteins) was added to 4-20\% SDS-PAGE gels, migration monitored at 150 volts for $1 \mathrm{~h}$, and the protein bands transferred to nitrocellulose membrane. The membrane was incubated with antibodies (anti-HIF- $1 \alpha$, anti- $\alpha$-tubulin antibody, anti-MMP2 antibody, anti-VEGF antibody, or anti-Glut 1 antibody) at $4^{\circ} \mathrm{C}$ for $16 \mathrm{~h}$ and labeled proteins detected with $0.1 \mu \mathrm{g} / \mathrm{mL}$ HRP-labeled anti-mouse IgG antibody (HIF-1 $\alpha /$ ab- 51608 or $\alpha$-tubulin/ab56676) or anti-rabbit IgG antibody (MMP2/ab52756, VEGF/ ab-52917 or Glut $1 / \mathrm{ab} 18106$ ) at $23^{\circ} \mathrm{C}$ for $1 \mathrm{~h}$. After ECL detection reagent development, the membranes were imaged with the FUJIFILM LAS 3000.

Chorioallantoic membrane assay

Chorioallantoic membrane experiments were performed as previously described [17]. Naïve U251-tumor biopsy fragments $\left(1-2 \mathrm{~mm}^{3}\right)$ were grafted onto the CAM, incubated with PBS-control or with a single bolus of either EZN-2208 or CPT-11. On day 12, the angiogenic response,
Left panels The percent of tumor bioluminescence remaining $48 \mathrm{~h}$ (gray) and $120 \mathrm{~h}$ (black) post-treatment compared to baseline (white). Right panels Images acquired at baseline and $120 \mathrm{~h}$ post-treatment. Graph depict mean \pm SEM luminescence normalized to group average $(* P<0.05 ; * * P<0.01)$

as determined by the number of vessels converging toward the grafts, was evaluated. CAMs were then processed for light- and dark-field microscopy, as previously described [18]. Microvessels density was expressed as the percentage of the total number of intersection points occupied by CD31-positive vessels cut transversely (diameter, 3-10 $\mu \mathrm{m})$ and mean values \pm SEM determined.

\section{Matrigel plug assay}

The matrigel angiogenesis assay was modified based on previously described methods $[19,20]$. In brief, matrigel (BD Biosciences) was prepared with or without $1 \mu \mathrm{g} / \mathrm{ml}$ basic-FGF (bFGF). Six week old female nude mice, which were anesthetized with Isoflurane, were then injected with matrigel subcutaneously in the ventral midline. No longer than 30 min following the implantation of matrigel, mice were administered intravenously a single dose of saline, EZN-2208 at $30 \mathrm{mg} / \mathrm{kg}$, or CPT11 at $80 \mathrm{mg} / \mathrm{kg}$. Six days later, the matrigel plugs were excised, photographed and 
weighed. Plugs were homogenized and analyzed for hemoglobin content using the Drabkin's assay according to manufacturer's instructions (Sigma D5941). A MannWhitney test was used to determine statistical significance.

\section{Histological analysis}

U251-HRE xenografts were administered, intravenously, a single dose of saline at the MTD of EZN-2208 (30 mg/kg) or CPT-11 $(80 \mathrm{mg} / \mathrm{kg})$. Five days after last treatment, the mice were sacrificed, tumors were collected, paraffin-embedded and stored until sectioned. Sections were de-paraffinized, rehydrated and processed for antigen retrieval as described before [21]. The sections were stained with a primary antibody against CD31 (clone SC-1506 Santa Cruz Biotechnology). Morphometric analysis for CD31-labeled areas was performed on nine randomly selected fields, within three separate sections, with an Image Analysis software-equipped Olympus photomicroscope (Olympus Italia) $(\times 200$ magnifications). The mean CD31-positive microvessels value \pm SEM of the nine fields per section and the final mean value for all three sections within a treatment group were calculated. Differences between means were determined by Student's $t$ test at $\mathrm{P} \leq 0.05$ (GraphPad Prism 3.0).

\section{Results and discussion}

EZN-2208 preferentially inhibits HIF-1-dependent luciferase expression compared with CPT-11

The U251-HRE cell line expresses a luciferase reporter controlled by a hypoxia response element that has shown up to 12-fold induction of in vitro luciferase expression under hypoxic $\left(1 \% \mathrm{O}_{2}\right)$ conditions $[10,22]$ and been used to investigate the HIF- $1 \alpha$ associated antitumor activity of topotecan by BL imaging [11]. We compared the efficacy of EZN-2208 and CPT-11, as single or multiple doses at their respective MTDs, to inhibit HIF-1-dependent luciferase expression and tumor growth in U251-HRE xenografts. It is important to note that since the PEG-component of EZN-2208 has no toxicity or antitumor activity, the activity of EZN-2208 is derived from SN38 that is released from the conjugate. In contrast, the cytotoxic activity of CPT-11 can be due to CPT-11 itself (the prodrug) as well as SN38 derived from CPT-11 [23]. Based on pharmacokinetic studies in mice, it is estimated that $1.5 \% \mathrm{SN}-38$ is derived from CPT-11 [14]. Since SN38 is approximately 100- to 1,000-fold more potent than CPT-11 [14], the activity of CPT-11 is derived from both drugs. The MTD of each of these compounds were chosen since our intent was to study the mechanistic basis of the maximal antitumor effect. At the MTDs, in either CPT-11 or EZN-2208 groups using a single or multidose schedule, no animal deaths were observed and body weight loss is routinely less than $5 \%$ pretreatment animal weight.

While the saline-treated mice had progressive increases in luminescence (data not shown), both the single and multiple EZN-2208-treatment groups had diminished luminescence at 48 and $120 \mathrm{~h}$ time points post Day 1 of treatment. CPT-11 treatment had minimal effect on luminescence by $48 \mathrm{~h}$, even following multiple doses (Fig. 1a, b-right panels). Because the tumor mass was reduced by both EZN-2208 and CPT-11 treatment (data not shown), the luminescence (total flux/photons/s) was normalized for tumor mass and expressed in terms of percent change from each respective group's baseline BL reading. A single dose of EZN-2208 induces potent and sustained down-regulation via the HIF- $1 \alpha$-luciferase regulated hypoxia response element ( $37 \%$ at $48 \mathrm{~h}$ and $83 \%$ at $120 \mathrm{~h}$ ) compared to no down-regulation by a single injection of CPT-11 (Fig. 1aleft panel). When given on multiple days (Day 0, 2, 4), EZN-2208 induces a very potent down-regulation of HIF$1 \alpha(38 \%$ by $48 \mathrm{~h}$ and $93 \%$ by $120 \mathrm{~h})$ (Fig. 1b-left panel) while CPT- 11 causes only a modest $15 \%(48 \mathrm{~h})$ and $32 \%$ $(120 \mathrm{~h})$ down-regulation. This observation agrees with Rapisarda et al., in which daily, but not intermittent, administration of topotecan, another topoisomerase I inhibitor, resulted in a sustained tumor growth inhibition [7]. Beyond this, since weight loss after administration of EZN-2208 or CPT-11 is mild (maximally 5 and $3 \%$ pretreatment weight, respectively) and transitory (recovery by day 5 or 6), while the down regulation of HIF- $1 \alpha$ occurs on day 2 and intensifies on day 5 , it is unlikely that the antiangiogenic effects are due an indirect effect of the compounds on weight loss. Thus, a continued delivery system of SN38 by EZN-2208 can down-regulate HIF-1 $\alpha$ in a more potent and sustained manner compared to CPT- 11 .

\section{EZN-2208 down-regulates mRNA levels of HIF- $1 \alpha$ targeted genes}

HIF-1 inhibitor therapy can target the HIF-1 pathway at several levels, including decreasing HIF-1 mRNA or protein levels or decreasing HIF-1-mediated trans-activation of downstream targets. To further confirm the role EZN2208 plays in the inhibition of the U251-HRE's HIF- $1 \alpha$ dependent luciferase expression and to determine the level of the inhibition, the intra-tumor HIF- $1 \alpha$ and downstream HIF-1-target (VEGF, Glut 1, Glut2, and TGF $\beta 1$ ) mRNAs were determined by RT-PCR, 48 and $120 \mathrm{~h}$ after a single injection (iv.) of either EZN-2208 $(30 \mathrm{mg} / \mathrm{kg})$ or CPT-11 $(80 \mathrm{mg} / \mathrm{kg}$ ) (Fig. 2a-f). These downstream targets play different roles in malignant cell survival. VEGF (VEGFR1) and MMP2 genes are involved in pro-angiogenesis and vascular stability, increasing under the hypoxic conditions. 

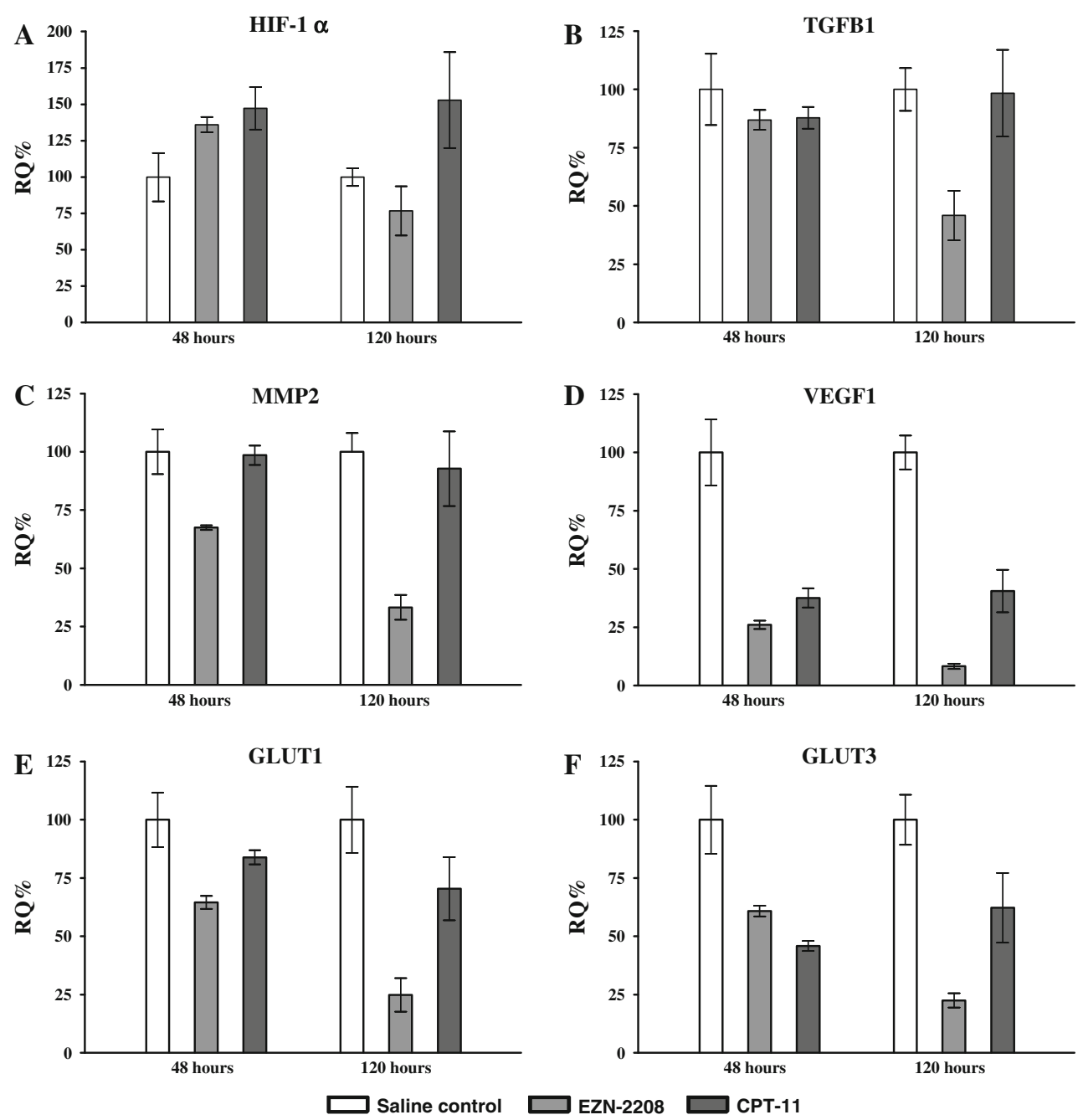

Fig. 2 RT-PCR analysis for mRNA expression in U251-HRExenograft tumors at 48 and $120 \mathrm{~h}$ after a single IV injection of EZN-2208 $(30 \mathrm{mg} / \mathrm{kg})$ or CPT-11 $(80 \mathrm{mg} / \mathrm{kg})$. mRNA RQ\% for

VEGF1, MMP2, GLUT1, GLUT3 and TGF $\beta 1$ from Saline control (white), EZN-2208 (gray) and CPT-11 (dark gray)

Glut1 and Glut3 play critical roles in cellular metabolism and glucose transport where enhanced glucose metabolism is observed following up-regulation of their respective genes by hypoxia. TGF $\beta 1$ stimulates cell proliferation [2, 24]. The stabilization of HIF- $1 \alpha$ proteins in the tumor's hypoxic environment, its translocation to the nucleus where it complexes with HIF-1 $\beta$, has been linked with the activation of these target genes that are important for cell survival under hypoxic conditions [24].

HIF- $1 \alpha$ mRNA levels (Fig. 2a) were not significantly changed (relative to vehicle-treated group) at $48 \mathrm{~h}$ and down-regulated slightly (20\%) at $120 \mathrm{~h}$ in U251-HRE xenograft tumors following a single injection of EZN-2208 $(30 \mathrm{mg} / \mathrm{kg}$ ) while no down-regulation by CPT-11 was observed. The results are consistent with those reported by Rapsirada et al., where topotecan did not affect HIF- $1 \alpha$ mRNA accumulation or protein half-life, but did inhibit its translation [12]. However, the single dose of EZN-2208, by

$120 \mathrm{~h}$ post-treatment, significantly down-regulated the mRNA of the downstream HIF- $1 \alpha$ targeted genes, including MMP2 (67\%), VEGF1 (92\%), Glut1 (75\%), Glut3 (77\%), and TGF $\beta 1$ (54\%) (Fig. 2b-f). Only VEGF1 mRNA levels were decreased by CPT-11 at both 48 (62\%) and $120 \mathrm{~h} \mathrm{(59 \% )} \mathrm{(Fig.} \mathrm{2d).} \mathrm{Glut1} \mathrm{and} \mathrm{Glut3,} \mathrm{were} \mathrm{slightly}$ down-regulated by CPT-11 at both 48 and $120 \mathrm{~h}$ (Glut1$16 \%$ and $30 \%$, Glut $3-54 \%$ and $38 \%$, respectively). The mRNA levels of all the other down-stream HIF- $1 \alpha$ targeted genes were unchanged following CPT-11 treatment, at both time points (Fig. 2e-f).

EZN-2208 down-regulates protein levels of HIF-1 $\alpha$, VEFG, Glut1 and MMP2

Increased HIF- $1 \alpha$ protein in cancer cells translocates to the nucleus, complexes with HIF- $1 \beta$ and activates multiple downstream-genes involved in angiogenesis (VEGF), 


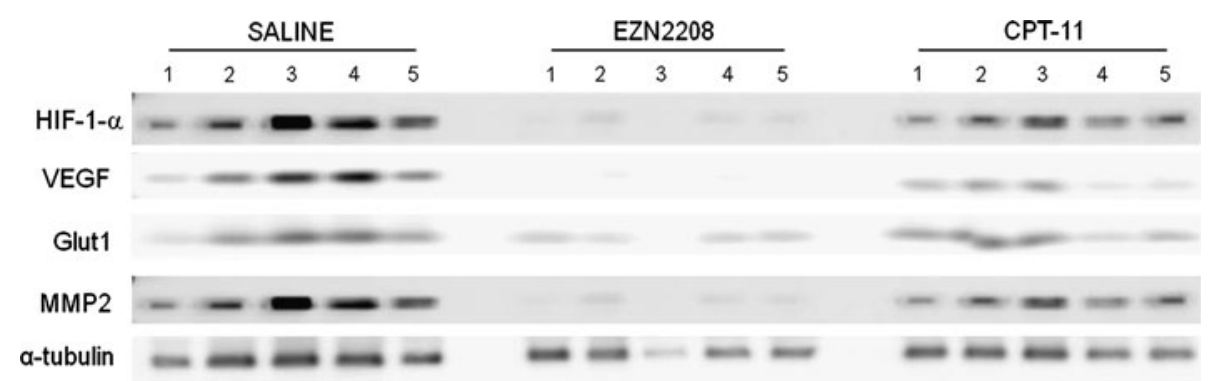

Fig. 3 Western blot analyses for HIF-1 $\alpha$, VEGF, Glut1, MMP2 and $\alpha$-tubulin expression in U251-HRE-xenograft tumors at $120 \mathrm{~h}$ after a single IV injection of EZN-2208 (30 mg/kg) or CPT-11 (80 mg/kg).
Protein expression levels in tumors isolated from individual mice, determined by SDS-PAGE electrophoresis
Fig. 4 Impact of EZN-2208 on angiogenesis by chorioallantoic membrane $(C A M)$ assay CAMs grafted from naive U251-HRE glioma tumor fragments were photographed in ovo with a camera-equipped stereomicroscope $(50 \times)$ following PBS, CPT-11 or EZN-2208 treatment as described. Images were taken using light and dark field microscopy as depicted in the upper and lower set of figures. Graph depicts the mean percent \pm SEM of CD31positive vessel density $(* * \mathrm{P} \leq 0.01 ; * * * \mathrm{P} \leq 0.001)$
A control
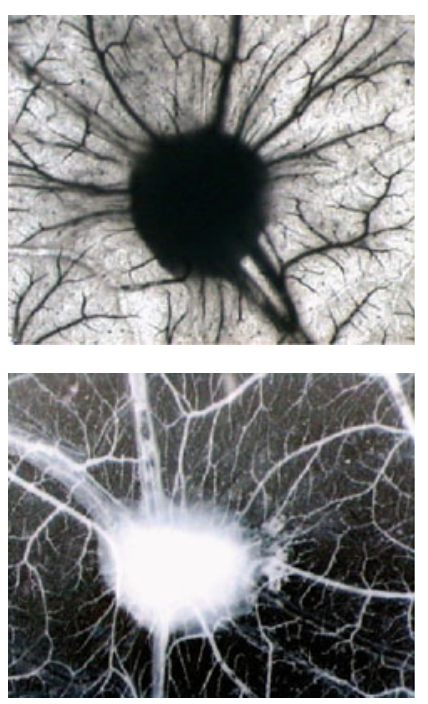

CPT-11

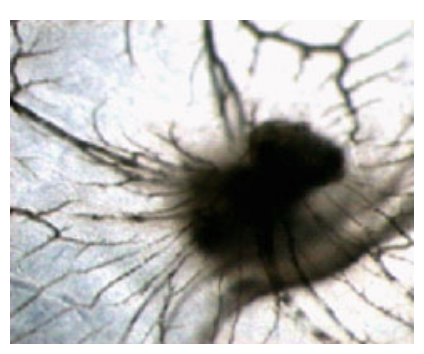

$E Z N-2208$
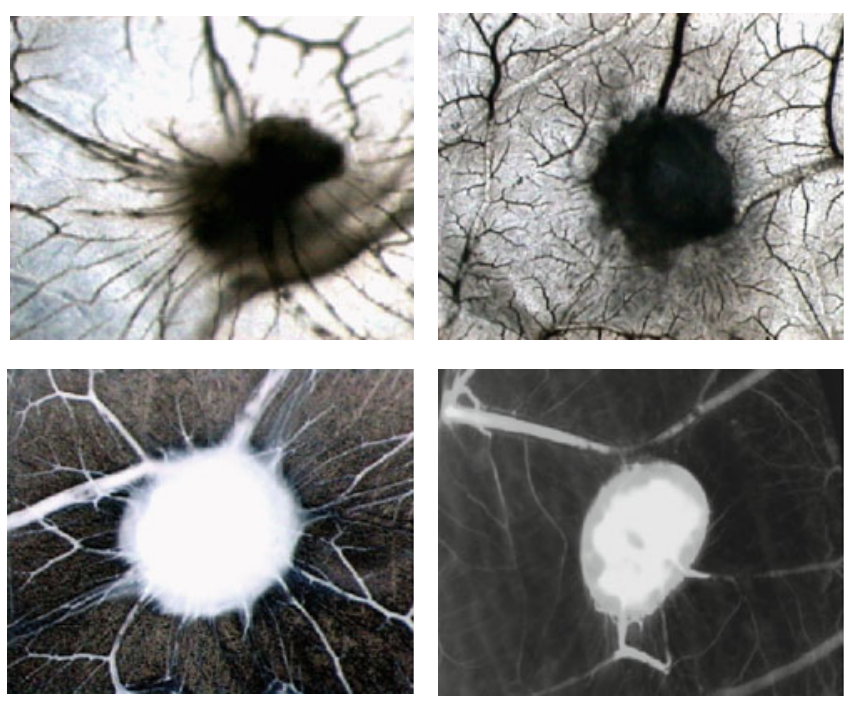

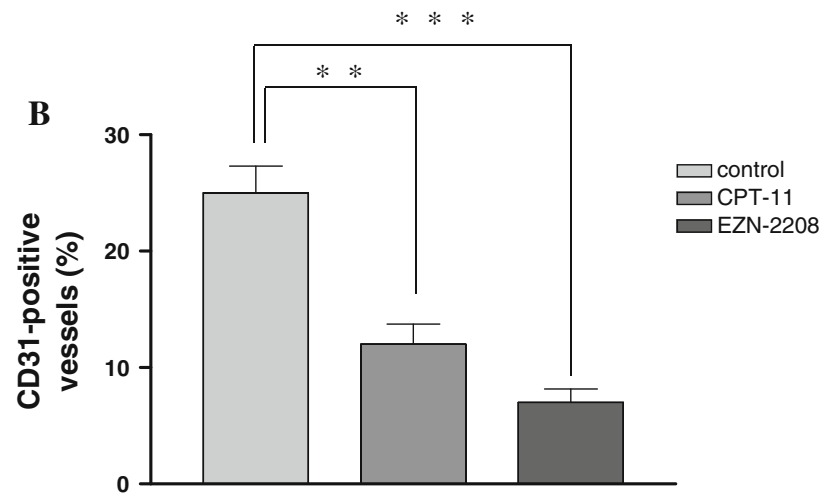

metabolic adaptation (Glut1), vascular stability and metastasis (MMP2) and, ultimately survival; thereby driving tumor progression [2, 24]. Western blot analyses (Fig. 3) of U251-HRE tumors from EZN-2208-treated mice indicated that EZN-2208 reduces the accumulation of HIF- $1 \alpha$ protein, significantly reducing the protein levels of VEGF, Glut1 and MMP2. Microtubule abundance (minimal $\alpha$-tubulin decrease detected) is not altered as a result of treatment. The tumors from mice similarly treated with CPT-11 showed a slight reduction in HIF-1 $\alpha$ protein levels and less effect on downstream VEGF and Glut1 protein levels as compared to EZN-2208. There was little effect on MMP2 or $\alpha$-tubulin levels with CPT11 treatment. 


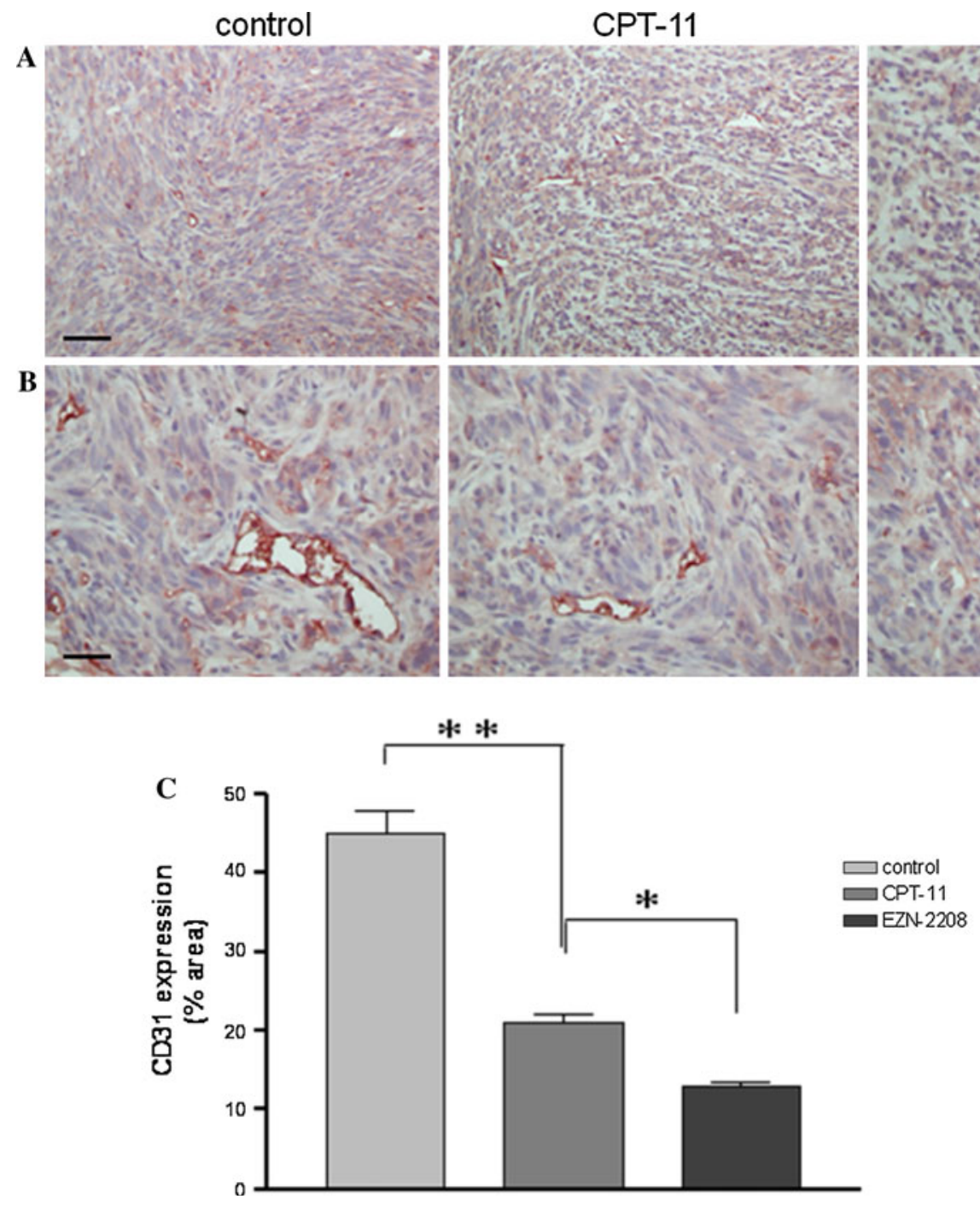

Fig. 5 IHC evaluation of CD31 expression levels in subcutaneous U251-HRE-derived xenograft tumors at low (a) and high magnification (b). Nude mice engrafted with U251-HRE cells, were injected with a single bolus of CPT-11 (80 mg/kg) or EZN-2208 $(30 \mathrm{mg} / \mathrm{kg})$.

Effect of EZN-2208 on angiogenic responses in the CAM assay, in tumors grown on the flank of nude mice, and the matrigel plug assay

To determine if the decreased VEGF protein levels observed after EZN-2208 treatment might improve antiangiogenic effects over that observed with CPT-11, the anti-angiogenic activity of EZN-2208 and CPT-11 was studied using the in vivo CAM assay. In this assay, antiangiogenic substances can cause differences in the extent and pattern of vessel branching following the implantation of the tumors, which are harvested from mice, and grown on the CAM in the presence of test agents. CAMs incubated with either CPT-11 or EZN-2208 showed a significant decrease in allantoic vessels radiating in a "spoked
Tumors were harvested 5 days after the treatment and tissue sections were immunostained for CD31 to show tumor angiogenesis. Scale bar 200 (a) and 100 (b) $\mu \mathrm{m}$. c Gaphs depict mean \pm SEM CD31 staining $(* P<0.05 ; * * P<0.01)$

wheel" pattern towards the xenografts compared to PBStreatment control CAMs (Fig. 4a, light and dark-field micrographs). Morphometric assessment of microvessel density also indicated that both compounds were effective, although EZN-2208 had a more pronounced effect (CPT-11 vs. control, $P<0.01$; EZN-2208 vs. control, $P<0.001$ ) (Fig. 4b).

The anti-angiogenic properties of CPT-11 vs. EZN2208 , as assessed by the amount of blood vessels in tumors treated with these agents was also compared. To do so, tumor expression of the blood vessel endothelial marker, CD31 was assessed using IHC methodology. It was found that both treatments exerted anti-angiogenic activities which resulted in decreased $\mathrm{CD} 31$ staining compared to PBS-treated tumors (Fig. 5) and however, the EZN-2208 
Fig. 6 Nude mice were implanted with matrigel containing bFGF or devoid of bFGF. Immediately after the implant, animals were given a single dose of saline, $30 \mathrm{mg} / \mathrm{kg}$ EZN-2208 or $80 \mathrm{mg} / \mathrm{kg}$ CPT-

11. Animals that received EZN2208 and CPT-11 had implants of matirgel plugs with $\beta$ FGF. After 6 days, matigel plugs were excised, photographed, and hemoglobin content/gm of plug weight was measured. a Graph depicts mean value $\pm \operatorname{SEM}(* P=0.0314 ; n s$ not significant). b Visual observation of excised matrigel implants

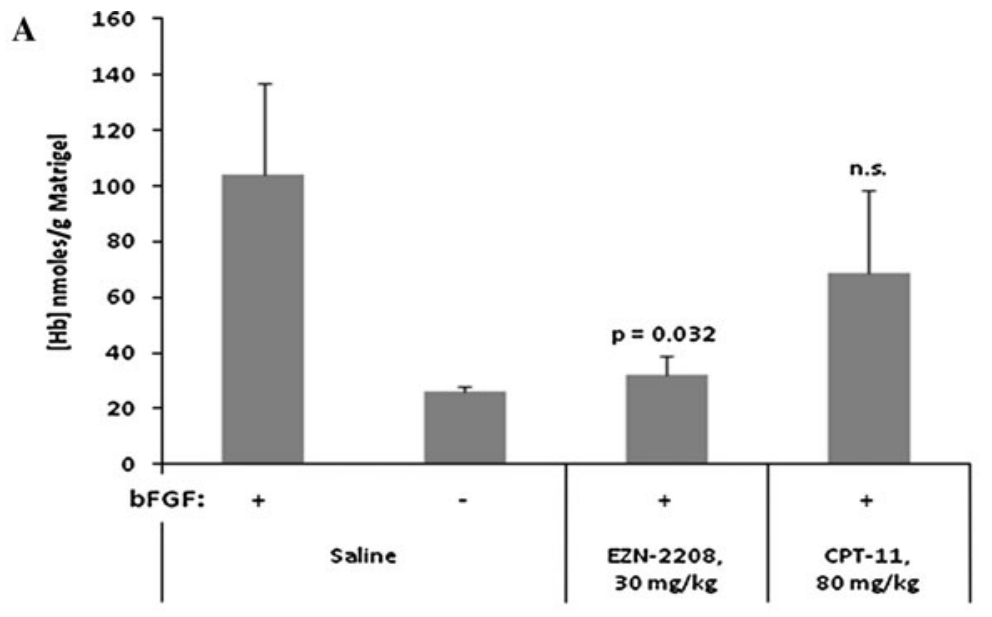

B

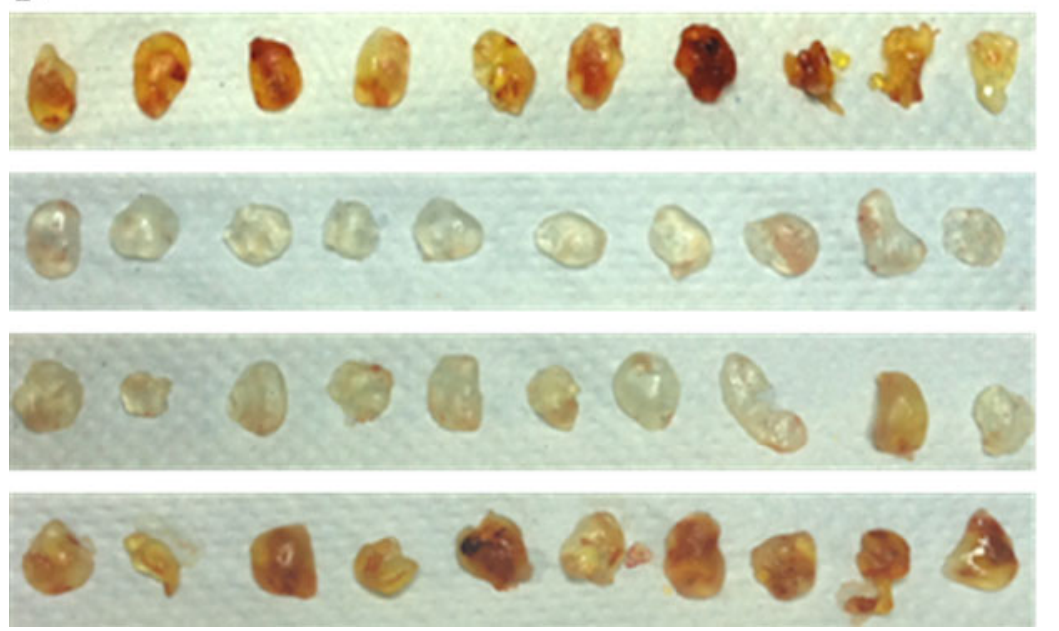

$+b F G F$

- bFGF

EZN-2208

CPT-11 treatment resulted in a significant increase in anti-angiogenic effect compared to CPT-11 $(P<0.05)$. Similar results have also been observed with tumors derived from neuroblastoma cell lines [16].

To further compare the anti-angiogenic properties of CPT-11 vs. EZN-2208, the ability of these compounds to inhibit the infiltration of blood vessels into a matrigel plug embedded with $\beta$ FGF was examined. Hemaglobin content was used to estimate amount of blood vessel that grew into the matrigel plug and has been previously validated as an acceptable assay [19]. Since no tumor cells were present in the matrigel plug, the effects of candidate anti-angiogenic effects are likely to be on endothelial cells. As shown in Fig. 6a, addition of $\beta$ FGF stimulated hemoglobin content in the plug by approximately fivefold and is consistent with previous reports [20]. EZN-2208 significantly reduced hemoglobin content in the $\beta$ FGF-embedded matrigel plug almost to the same values in matigel plugs devoid of bFGF. In contrast, CPT-11 had no significant effect. The conclusions were consistent with visual observation of the matrigel plugs (Fig. 6b).

\section{Conclusions}

In conclusion, data presented here and previously published by Mellilo et al. [6-8], strongly argues for sustained inhibition of HIF- $1 \alpha$ to achieve therapeutically meaningful responses in preclinical models. Clinical trials sponsored by the NCI are ongoing to explore the hypothesis that chronic administration of topotecan inhibits HIF- $1 \alpha$ expression and angiogenesis in patients with metastatic tumors over-expressing HIF-1 $\alpha$ (www.clinicaltrials.gov). Our data argues that high and sustained SN38 delivery by EZN-2208 down-modulate HIF- $1 \alpha$ protein and further downstream regulated genes and proteins, in particular VEGF, in a long lasting manner. This mechanism, along with direct effects on the tumor vasculature, may contribute to improved efficacy of EZN-2208 over CPT-11 that has been observed in several preclinical xenograft models, including CPT-11 refractory tumor models [14].

Acknowledgments The authors would like to thank Dr. G. Melillo and A. Rapisarda for providing the U251-HRE cell line and for their expert technical assistance. 
Competing interests All Enzon employees are or were full time employed at Enzon at time studies were done and own company stock/stock options. Notes: Dr. Pastorino received a research grant from Enzon for their collaboration.

Open Access This article is distributed under the terms of the Creative Commons Attribution Noncommercial License which permits any noncommercial use, distribution, and reproduction in any medium, provided the original author(s) and source are credited.

\section{References}

1. Semenza GL (2000) Hypoxia, clonal selection, and the role of HIF-1 in tumor progression. Crit Rev Biochem Mol Biol 35: 71-103

2. Semenza GL (2003) Targeting HIF-1 for cancer therapy. Nat Rev Cancer 3:721-732

3. Zhong H, De Marzo AM, Laughner E et al (1999) Overexpression of hypoxia-inducible factor 1alpha in common human cancers and their metastases. Cancer Res 59:5830-5835

4. Rahman R, Smith S, Rahman C et al (2010) Antiangiogenic therapy and mechanisms of tumor resistance in malignant glioma. J Oncol 251231 (Epub 2010 Apr 11)

5. Takano S, Yamashita T, Ohneda O (2010) Molecular therapeutic targets for glioma angiogenesis. Published online J Oncology 351908 (Epub 2010 Apr 18)

6. Nussenbaum F, Herman IM (2010) Tumor angiogenesis: insights and innovations. J Oncol 132641 (Epub 2010 Apr 26)

7. Ferrara N, Gerber HP, LeCouter J (2003) The biology of VEGF and its receptors. Nat Med 9:669-676

8. Semenza GL (2007) Evaluation of HIF-1 inhibitors as anticancer agents. Drug Discov Today 12:853-859

9. Poon E, Harris AL, Ashcroft M (2009) Targeting the hypoxiainducible factor (HIF) pathway in cancer. Expert Rev Mol Med 11:e26

10. Rapisarda A, Yranchimeg B, Scudiero DA et al (2002) Identification of small molecule inhibitors of hypoxia-inducible factor-1 transcriptional activation pathway. Cancer Res 62:4316-4324

11. Rapisarda A, Zalek J, Hollingshead M et al (2004) Scheduledependent inhibition of hypoxia-inducible factor-1 protein accumulation, angiogenesis, and tumor growth by topotecan in U251-HRE glioblastoma xenografts. Cancer Res 64:6845-6848

12. Rapisarda A, Uranchimeg B, Sordet O et al (2004) Topoisomerase I-mediated inhibition of hypoxia-inducible factor-1: mechanism and therapeutic implications. Cancer Res 64:1475-1482

13. Zhao H, Rubio B, Sapra P et al (2008) Novel prodrugs of SN38 using multiarm poly(ethylene glycol) linkers. Bioconjugate Chem 19:849-859

14. Sapra P, Zhao H, Mehlig M et al (2008) Novel delivery of SN38 markedly inhibits tumor growth in xenografts, including a Camptothecin-11 refractory model. Clin Cancer Res 14:1888-1896

15. Sapra P, Kraft P, Mehlig M et al (2009) Marked therapeutic efficacy of a novel polyethylene glycol-SN38 conjugate, EZN2008 , in xenograft models of non-Hodgkin's lymphoma. Hematologica 10:1456-1459

16. Pastorino F, Loi M, Sapra P et al (2010) Tumor regression and curability of preclinical neuroblastoma models by PEGylated SN38 (EZN-2208), a novel topoisomerase I inhibitor. Clin Cancer Res 16:4809-4821

17. Ribatti D, Nico B, Vacca A et al (2006) The gelatin spongechorioallantoic membrane assay. Nat Protoc 1:85-91

18. Pastorino F, Paolo DD, Piccardi F et al (2008) Enhanced antitumor efficacy of clinical-grade vasculature-targeted liposomal doxorubicin. Clin Cancer Res 14:7320-7329

19. Pastorino F, Brignole C, Di Paolo D et al (2006) Targeting liposomal chemotherapy via both tumor cell-specific and tumor vasculature-specific ligands potentiates therapeutic efficacy. Cancer Res 66:10073-10082

20. Passanti A, Taylor RM, Pili R et al (1992) A simple, quantitative method for assessing angiogenesis and antiangiogenesis using reconstituted basement membrane, heparin, and fibroblast growth factor. Lab Invest 67:519-528

21. Kumar R, Knick VB, Rudolph SK et al (2007) Pharmacokineticpharmacodynamic relationship from mouse to human with pazopanib, a multikinase angiogenesis inhibitor with potent antitumor and antiangiogenic activity. Mol Cancer Thera 6:2012-2021

22. Melillo G (2007) Hypoxia-inducible factor 1 inhibitors. Methods Enzymol 435:385-402

23. Mathijssen RHJ, van Alphen RJ, Verweij J et al (2001) Clinical pharmacokinetics and metabolism of irinotecan (CTP-11). Clin Cancer Res 7:2182-2194

24. Rankin EB, Giaccia AJ (2008) The role of hypoxia-inducible factors in tumorigenesis. Cell Death Differ 15:678-685 